\title{
Design and Implementation of Kogge Stone adder using CMOS and GDI Design: VLSI Based
}

\author{
S.Vasantha swaminathan, J.Surendiran, B.P.Pradeep kumar
}

\begin{abstract}
Unique-Adders is a significant part in different math legitimate activity. Parallel Prefix Adder was developed as the most basic and effective circuit for double expansion. The Particular structure and execution are alluring for VLSI usage. In these papers, I can depict the structure and execution of the Kogge Stone Parallel Prefix Adders and actualized utilizing diverse plan procedure. CMOS (Complementary Metal Oxide Semiconductor) and GDI (Gate Diffusion Input) are the distinctive structure system utilized. . The plan and reenactment of rationale entryways is performed on CADENCE Design Suit 6.1.6 utilizing virtuoso and ADE Environment at GPDK 180nm innovation. The execution estimation considered for the presentation of the KSA is delay, number of door check/Transistor Count (territory) and power. Recreation reads are accomplished for 4-piece, 8-piece and 16-piece input information.
\end{abstract}

Catchphrases-Parallel Prefix Adder, Kogge Stone Adder, CMOS plan, GDI structure, Cadence Design Suite, 180nm innovation, Area, Power, Delay and Power Delay Product.

Catchphrases : About four watchwords or expressions in sequential order request, isolated by commas.

\section{INTRODUCTION}

The expansion of two paired numbers is one of the most fundamental square and significant job in the math rationale work in current advanced frameworks, for example, microchip and computerized signal preparing. These frameworks paired adders are significant parts of on Arithmetic and Logic Unit (ALU) of CPU . In VLSI framework plan, the prerequisites of viper ought to be quick and effective in term of intensity, speed (less postponement) and territory. These presentation tasks principally rely upon snake plan engineering. In this paper consider the distinctive degree of Kogge Stone Parallel Prefix Adder is structured utilizing diverse innovation CMOS (Complementary Metal Oxide Semiconductor) and GDI (Gate Diffusion) rationale and near examination of the adders are arranged.

The noteworthy issue for the parallel expansion is the spread deferral in the convey chain. On the off chance that the information bit or operand expands the length of convey affix likewise increments To dodge this issue of Carry chain spread, present day Parallel Prefix Adders design is utilized, it comprising of Pre-Processing, Carry Look-Ahead (Carry Generate) and Post-Processing segments.

The adders are set up on most proficient circuit for each parallel expansion in advanced frameworks, execution and postponement of these adders principally relies upon number of levels in the convey produced organize.

\section{LITERATURE REVIEW}

Coming up next are the couple of reference that I have experienced for the parallel prefix adders structure, usage and result. Plan and execution of the various adders like RCA, CSA, CLA and KSA. give a short working of plan and execution of 8-piece Kogge Stone Adder (KSA) and its changed KSA configuration utilizing rhythm apparatus. The Brief working of Parallel Prefix Adders and execution of six distinctive parallel prefix adders structure and usage utilizing diverse TSNC innovation hubs, they are KSA, BKA, HCA, Sklansky Adder, LFA and Knowel Adder. Relative examination of various Kogge Stone Adders with Ripple Carry and Carry Skip Adders.

The Frontend plan and execution various adders like KSA and BKA. The detail depiction and working about GDI (Gate Diffusion Input) innovation, The detail portrayal and working of CMOS (Complementary Metal Oxide Semiconductor) Technology.

\section{DESIGN OF KOGGE STONE ADDER}

The Kogge Stone Adder is a Parallel Prefix type of Carry Look-Ahead Adder. It creates the convey signal in O ( $\log 2$ $\mathrm{N})$ time. This snake is broadly utilized in the business and considered as the quickest viper structure. Adders are produced quick by registering them in parallel, speed of activity is extremely high because of the low profundity of hub and activity done in parallel and principle significant factor is the result of the snake is rely on the underlying sources of info. A three stage process is by and large engaged with the development of a Kogge Stone Parallel Prefix Adder

\footnotetext{
Revised Manuscript Received on October 22, 2019.

Dr.S.Vasantha swaminathan, Professor, Dept. of ECE, Malla Reddy Engineering College for Women(Autonomous), Secunderabad-TS vasanthmrecw@gmail.com

J.Surendiran, Professor, Dept. of ECE, HKBHCE, Bangaluru surenjaya1981@gmail.com

B.P.Pradeep Kumar, Associate Professor, Dept. of ECE, HKBHCE, Bangaluru,pradi14cta@gmail.com
} 


\section{Design and Implementation of Kogge Stone adder using CMOS and GDI Design: VLSI Based}

\section{RESULT AND DISCUSSIONS}

Reenactment Results

Coming up next are the schematic and recreation aftereffects of 4-piece, 8-piece and 16-piece Kogge Stone Parallel Prefix adders and structure usage done utilizing diverse plan Technique.(CMOS,GDI and CMOS-GDI rationales).

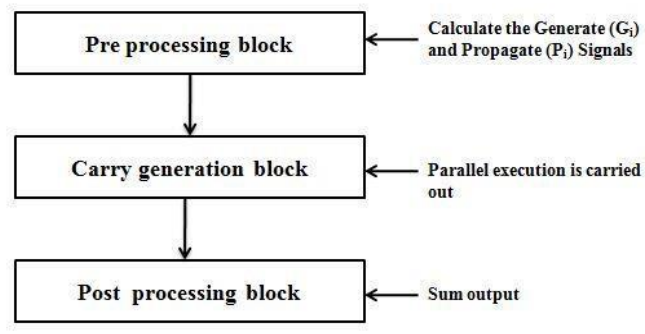

Figure 1: Architecture of Kogge Stone parallel prefix adder.

Schematic and Simulation of 8-bit KSA using GDI Comparative Analysis

The Kogge Stone Adder designed with different design techniq gate/transistor count (area), delay and power. The result is shown in

Table 1: Comparative analysis of 4-bit KSA adder

\begin{tabular}{|c|c|c|c|}
\hline \multicolumn{2}{|c|}{$\begin{array}{l}\text { Performance } \backslash \mathrm{KSA} \\
\text { Adder }\end{array}$} & $\begin{array}{l}\text { CMOS } \\
\text { Design }\end{array}$ & $\begin{array}{l}\text { GDI } \\
\text { Design }\end{array}$ \\
\hline \multirow{5}{*}{ Delay (ns) } & $\mathrm{s} 0$ & $11.22 \mathrm{e}-9$ & $11.06 \mathrm{e}-9$ \\
\hline & s1 & $11.22 \mathrm{e}-9$ & $11.94 \mathrm{e}-9$ \\
\hline & $\mathrm{s} 2$ & $21.54 \mathrm{e}-9$ & $31.27 \mathrm{e}-9$ \\
\hline & s3 & $31.43 \mathrm{e}-9$ & $44.02 \mathrm{e}-9$ \\
\hline & Cout & $\begin{array}{l}41.4 \\
3 e-9\end{array}$ & $\begin{array}{l}90.9 \\
5 e-9\end{array}$ \\
\hline \multicolumn{2}{|c|}{$\begin{array}{l}\text { Gate Count/ Number } \\
\text { of } \\
\text { transistors }\end{array}$} & $40^{2}$ & 8 \\
\hline \multicolumn{2}{|l|}{ Power (W) } & $\begin{array}{l}1.3493 \\
4 \mathrm{e}-5\end{array}$ & $\begin{array}{l}3.3624 \\
0 e-7\end{array}$ \\
\hline
\end{tabular}

Table 2: Comparative analysis of 8-bit KSA adder

\begin{tabular}{|c|c|c|c|c|}
\hline $\begin{array}{c}\text { Perforı } \\
\text { Design } \\
\text { Style }\end{array}$ & & $\begin{array}{l}\text { CM } \\
\text { OS } \\
\text { Desi } \\
\text { gn }\end{array}$ & $\begin{array}{l}\text { GDI } \\
\text { Design }\end{array}$ & $\begin{array}{l}\text { Modifi } \\
\text { ed } \\
\text { GDI }\end{array}$ \\
\hline & s0 & $\begin{array}{c}11.2 \\
2 \mathrm{e}-9\end{array}$ & ${ }_{-9}^{11.06 \mathrm{e}}$ & $9^{21.07 \mathrm{e}-}$ \\
\hline & s1 & $\begin{array}{c}11.2 \\
2 \mathrm{e}-9\end{array}$ & $9^{12.06 \mathrm{e}}$ & $9^{32.06 \mathrm{e}-}$ \\
\hline & s2 & $\begin{array}{c}21.4 \\
5 e-9\end{array}$ & ${ }_{-9}^{31.31 \mathrm{e}}$ & $9^{33.14 \mathrm{e}-}$ \\
\hline $\begin{array}{l}\text { Delay } \\
\text { (ns) }\end{array}$ & s3 & $\begin{array}{l}31.4 \\
5 \mathrm{e}-9\end{array}$ & ${ }^{33.03 \mathrm{e}}$ & $9^{49.76 \mathrm{e}-}$ \\
\hline & s4 & $\begin{array}{c}41.4 \\
5 \mathrm{e}-9\end{array}$ & ${ }_{-9}^{41.25 \mathrm{e}}$ & $9^{52.13 e-}$ \\
\hline & s5 & $\begin{array}{l}51.5 \\
5 \mathrm{e}-9\end{array}$ & $a^{52.76 e}$ & $9^{63.88 e-}$ \\
\hline & s6 & $\begin{array}{c}61.5 \\
5 \mathrm{e}-9\end{array}$ & ${ }_{-9}^{65.03 e}$ & $9^{72.68 \mathrm{e}-}$ \\
\hline & s7 & 71.4 & $74,02 \mathrm{e}$ & $82.29 \mathrm{e}-$ \\
\hline
\end{tabular}

The paper gives the plan and execution of Kogge stone viper utilizing different strategies as 4-piece, 8-piece, and 16-piece. The Adders are planned and actualized utilizing Cadence Design Suite at GPDK 180nm innovation, from the similar examination we can perceive how the presentation can change from 4 piece to 16 piece viper engineering. Kogge Stone Adder is planned utilizing three diverse structure methods like CMOS and GDI strategy.

\section{REFERENCES}

. Kunjan D. Shinde, Jayashree C. N. "Modeling, Design and Performance Analysis of Various 8-bit Adders for Embedded Applications", Proceedings of the ELSEVIER in International Conference on Emerging

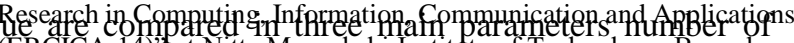
(ERCICA-14)' at Nitte Meenakshi Institute of Technology, Bangalore,

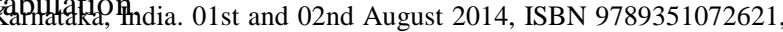
pp. 823 to 831 .

2. Nehru, K., \& Shanmugam, A. (2015). Analysis of 16-bit counter using GDI technique and CMOS logic. Int. J. Appl. Eng. Res, 10(6), 16121-16128.

3. Nehru, K., Shanmugam, A., \& Vadivel, S. (2012, February). Design of 64-bit low power parallel prefix VLSI adder for high speed arithmetic circuits. In Computing, Communication and Applications (ICCCA), 2012 International Conference on (pp. 1-4). IEEE

4. Nehru, K., \& Shanmugam, A. (2012). Design of 64 Bit Parallel Prefix Adder using Transmission Gate. European Journal of Scientific Research, 90(4), 593-605.

5. David H K Hoe, Chrix Matinez and Sri Jyothsna Vandavalli, "Design and Characterization of Parallel Prefix Adders Using FPGA",System theory (SSST), 2011 IEEE Hard South system

6. Sunder Kumar Yezerla, B Rajendra Naik, "Design and Estimation of Delay, Power and Area of Parallel Prefix Adders", Proceeding of 2044 RAECS UIET Punjab University Chandigad March 2014.

7. Kandasamy, N., Ahmad, F., \& Telagam, N. (2018). Shannon logic based novel qca full adder design with energy dissipation analysis. International Journal of Theoretical Physics, 57(12), 3702-3715.

8. N. Nithiyanandam, K. Venkatesh, M. Rajesh, Transfer The Levels Of The Monitored Carbon, Nitrogen Gases From The Industries, International Journal of Recent Technology and Engineering, Volume-7 Issue-6S3 April, 2019.

9. Sivanesh Kumar, A., Brittoraj, S., Rajesh, M., Implementation of RFID with internet of things, Journal of Recent Technology and Engineering, Volume-7 Issue-6S3 April, 2019.

10. Rajesh, M., Sairam, R., Big data and health care system using mlearningJournal of Recent Technology and Engineering, Volume-7 Issue-6S3 April, 2019.

11. Rajesh, M., and J. M. Gnanasekar. "Path Observation Based Physical Routing Protocol for Wireless Ad Hoc Networks." Wireless Personal Communications 97.1 (2017): 1267-1289. 